\title{
DEVELOPMENT OF A PROTOCOL FOR SCREENING LEUKEMIA FROM THE MICROSCOPIC IMAGES ACQUIRED FROM BLOOD SMEAR
}

\author{
Sanath Kumar Nayak ${ }^{1}$, Niranjana Sampathila ${ }^{2}$ \\ ${ }^{I}$ PG Scholor, Department of Biomedical Engineering, Manipal Institute of Technology, Manipal University, Manipal, \\ India \\ ${ }^{2}$ Associate Professor (Sr. Scale), Department of Biomedical Engineering, Manipal Institute of Technology, Manipal \\ University, Manipal, India
}

\begin{abstract}
Leukemia is a cancer, generally, leukemia is caused due to the over production of abnormal white blood cells (WBC). However, the abnormal white blood cells do not function the same way that the normal white cell does. These abnormal cells enter the blood stream and spreads throughout the body. Generally the detection of leukemia is done manually using a manual microscope by observing the white blood cells. The manual process consumes a lot of time and most of the times it leads to inaccurate result by faulty detection due to human error. As in remote areas, diagnosis of certain disease like leukemia is a challenging task as it requires a sophisticated lab setup with good clinical experts. The developed system using a USB digital Microscope captures the image from the blood smear with 40x magnification. Raspberry pi3 module is used for processing these captured images and later they are displayed on a monitor screen. Thus the developed hand held device along with image processing plays a major role in modern digital tele-pathology, and are expected to help in screening of blood cells in the remote area.
\end{abstract}

Keywords — Leukemia, Red Blood Cells, White Blood Cells, Acute Lymphoblastic Leukemia $* * *$

\section{INTRODUCTION}

Telemedicine literally means treating patients at a distance. The back support of this telemedicine is the internet, cell phones and computers. This service has revolutionized how an individual interact and communicate with another individual, how they seek information and exchange it with each other and improve their life. This is where the Information and Communication Technology (ICT) plays a major role in the field of healthcare [1].

Figure.1 shows optical microscope with digital camera, which is also called as light microscope. It uses visible light and a lens or series of lenses to magnify the components present in a blood smear. The digital microscope shown in the Figure 1 uses a lens very close to the viewing object. A high power camera is mounted on the microscope. When the light is focused on the real image, the components present on the slide gets enlarged using objective lens. The obtained image is further magnified by the eyepiece (ocular lens) that gives a magnified inverted virtual image of the focused object [2]. Thus the combination of objective lens and eyepiece will fetch an image with high magnification. A source of visible light is focused on a blood smear using condenser lens. This lens is placed at a certain distance below from the slide on a microscope. The light that is transmitted through the slide is received by the objective lens and it is magnified at a particular magnification power up to $100 x$. Thus, the objective lens produces a primary image of the viewed area. The ocular lens at the eyepiece usually has a magnification power of 10x. The primary image focused on the eyepiece gets magnified ten times, when the image is viewed by the observer [2].

The procedure of counting blood cells is done manually in all the laboratories. A special counter is required to count the cells present in the blood smear. This device is used to interpret the type of disease based on the count of particular cell.

In clinical diagnosis, this digital microscope plays a major role in the diagnosis of various pathological conditions such as malaria, leukemia and in other areas. The main focus of this work is to develop a hand held device to screen leukemia.

Considering the case of patients at remote area, the digital pathology allows pathologist to work remotely to deliver accurate results faster than traditional method. Generally, in most of the diagnostic centers and hospitals, leukemia diagnosis is done manually using a microscope. In manual process, the possibility of faulty detection due to human error may arise. In order to avoid these errors and to have highly accurate result, there is a need of excellent lab setup and clinical experts, which may be hardly found in the rural areas. In such situation, tele-pathology would be helpful for the patients visiting clinics in rural area [3]. 


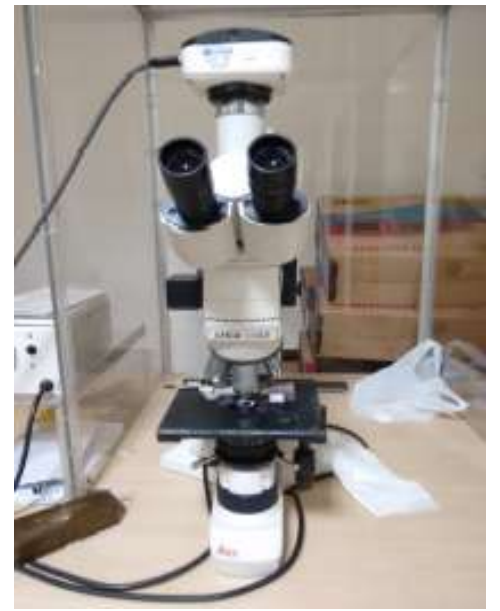

Fig 1: Digital Microscope.

Courtesy: Audio-Visual Department,. Manipal University

Leukemia is a cancer, that is developed by the over production of white blood cells as shown in Figure 2. It is produced in the blood forming stem cells that divide to reproduce more immature cells that get mature with time. The region for this development is mainly the bone marrow, the spongy tissue found within the bone. These white blood cells are responsible for body's immune response. The white blood cell production is in such a way that it replaces the population of the normal cells and the marrow cells/stem cells. Stem cells emerge from the bone marrow. With time, it will mature as myeloid stem cell or a lymphoid stem cell [4].

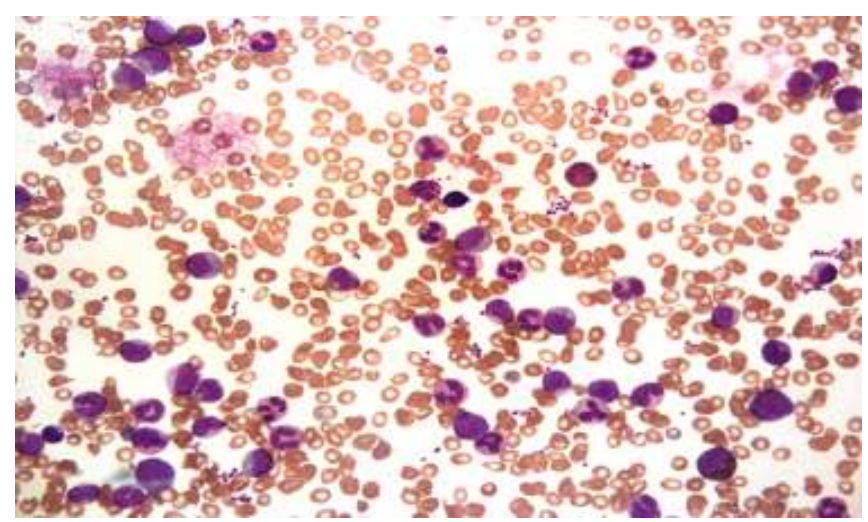

Fig 2: Over produced white blood cells.

Mainly there are two major categories of leukemia i.e. acute leukemia and chronic leukemia.

1. Acute Leukemia - it rapidly progresses and is detected at the initial leukemia stage. If it is not treated at the right time it may be dangerous. Standard treatment such as bone marrow transplant is done to cure this disease, especially in younger patients. The sub stages of acute leukemia are: Acute Lymphoblastic Leukemia (ALL) and Acute Myeloid Leukemia (AML) [5].

2. Chronic Leukemia - it is a slowly progressing disease. It can only be treated and managed but cannot be cured with any standard treatments. It is mostly seen in adults and elderly people. The sub stages of acute leukemia are:
Chronic Lymphocytic Leukemia (CLL) and Chronic Myeloid Leukemia (CML) [6,7].

\section{METHODOLOGY}

The Tele-device will serve people at remote area to get a primary diagnostic result. This system requires good internet facility and people with basic clinical and pathological skills. The system will be used to screen for the presence of leukemia in the blood image. The Tele-system shown in Figure 3 has three major blocks i.e. image acquisition, processing unit and leukemia screening.

\begin{tabular}{|c|c|c|}
\hline Image \\
\cline { 3 - 3 }
\end{tabular}$\longrightarrow \begin{gathered}\text { Processing } \\
\text { Unit }\end{gathered} \longrightarrow \begin{gathered}\text { Leukemia } \\
\text { Screening }\end{gathered}$

Fig 3: Block diagram of the Tele Device to Screen Leukemia.

\subsection{Image Acquisition}

The microscopic images were obtained using a hand held USB microscope device. This device is of a lesser magnification, approximately at the range of 40x resolution. This hand held microscope has a USB connector at one fixed end, which is connected to the USB port of the processing unit. The smear has three regions i.e. head, body and tail. Usually, the microscope is placed at the body region so that, there appears clear view of all the cells present in the blood. Due to lighting effect, initially, the image looks blurred on the screen. Therefore, the microscope has a manual focusing knob which gives the focused image.

\subsection{Processing Unit}

The processing unit in this proposed work uses a Raspberry $\mathrm{Pi} 3$ which is a compact open source board. Image that has been captured by the USB digital microscope contains raw image which is very difficult to process directly. The purpose of pre-processing is to remove the unwanted components present in the raw image and preserve the meaningful components.

For example, in the case of leukemia screening, the raw blood image contains RBC's, WBC's, Platelets, Parasites, noise and other artifacts. Thus, WBC's that are present in the blood image are preserved and the unwanted data are removed in pre-processing.

With an image processing approach, signatures of leukemia are obtained from the captured images, which are in the RGB color format. There are different color spaces for representing RGB image and among them some color spaces significantly express the signature [7].

Initially, in this work RGB image is converted in HSV color space. In the next step of processing, the image in HSV space is slit into Hue, Saturation, and Value components and then the threshold value is applied to Hue and Saturation components. 


\subsection{Screening of Leukemia}

In this stage, the processed image contains only WBC cells. Some of the WBC cells will be nucleated and the remaining WBC cells stay normal. These cells have different shapes, generally appears in circular form with uneven boundary. The original image is processed to get the segmented WBC.

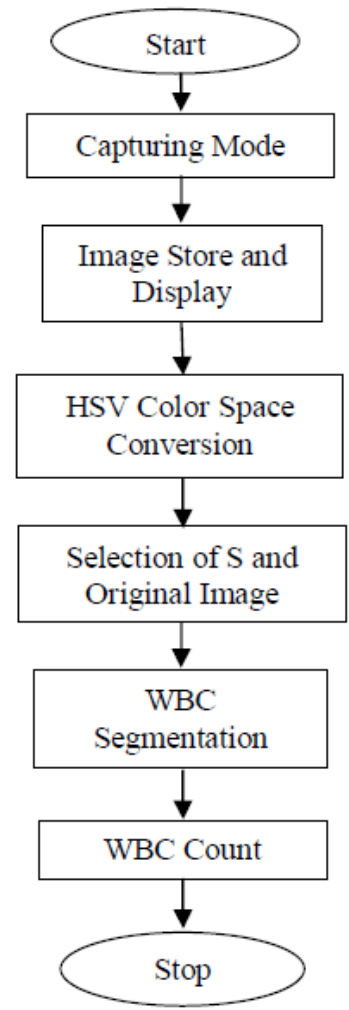

Fig 4: Flowchart of the proposed method.

\section{RESULT}

The project is aimed to design a hand held screening device which can help the physicians to check for the presence of leukemia from the microscopic blood image. Initially, the images from the digital microscope are considered and the total white blood cells present in a smear are calculated. The implemented algorithm is shown in Figure 4. The raspberry pi3 module and a hand held USB microscope are used to acquire the leukemia images from blood smear. In this area, the results obtained are presented after processing the original image. The WBC count is calculated using the automated approach, and it is then compared with the gold standard (machine count) and traditional method.

\subsection{Microscopic Image Analysis}

The images of different classes of leukemia, which are viewed using microscope is shown in the Figure 5. In this work only two cases are considered. The samples of one normal blood smear and one smear of acute lymphoblastic leukemia are taken. In each smear sample, there are three regions i.e. head, body and tail as shown in the Figure 6. The most prefered area for analysis is the body region i.e. between the head and the tail region. It is because the cells will be uniformly distributed in this region.
From these considered samples, the cells are counted manually in ten fields by viewing the blood slide through a microscope under 400x magnification. Later, the mean value of number of cells per field is calculated. Then, the standard multiplication factor is applied to obtain a total WBC count.

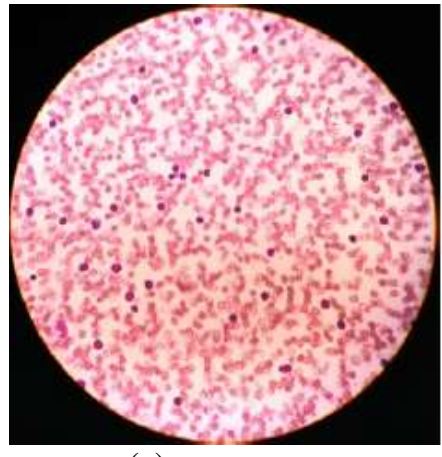

(a)

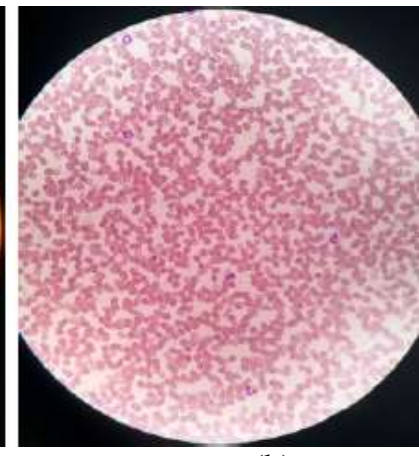

(b)
Fig 5: Microscopic images of blood smears. (a) Microscopic image of Acute Lymphoblastic Leukemia captured from phone. (b) Microscopic image of a normal blood sample captured from phone.

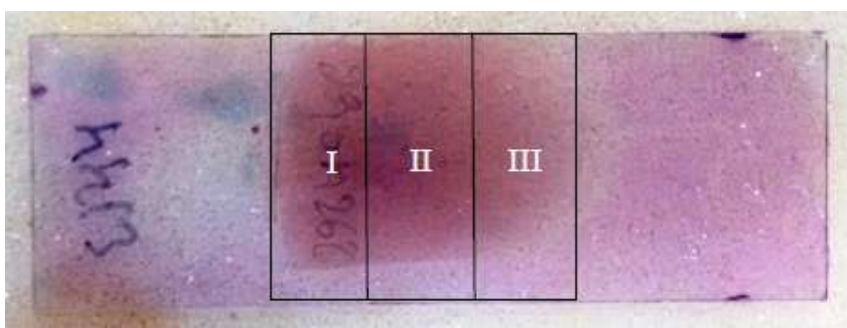

Fig 6: Slide with blood smear. (I) Head Region. (II) Body Region. (III) Tail Region.

\subsection{Automated Analysis}

The image shown in Figure 7(a) is of ALL. It displays the number of white blood cells that is present in the blood smear. To count the total number of WBC cells present in the entire smear, ten more images are considered and processed. The mean values of ten images are taken and then the multiplication factor was applied to obtain the total WBC count. Figure 7(b, d) shows the resultant image with the count value printed on one image. Similarly, ten more images undergo same procedure in each of the case.

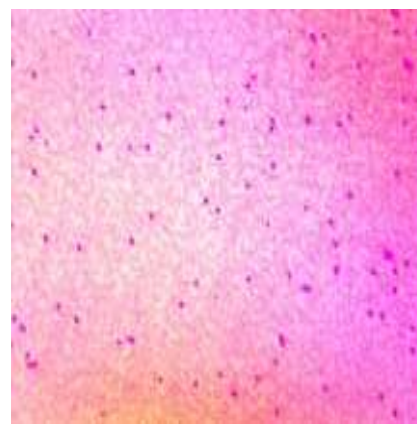

(a)

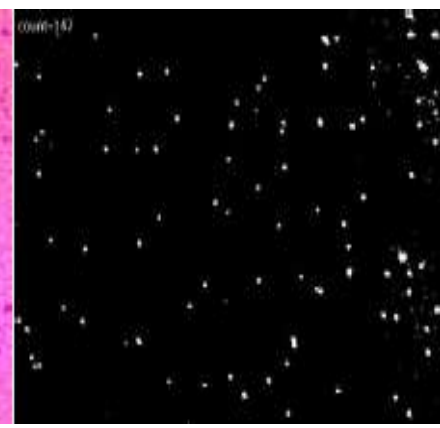

(b) 


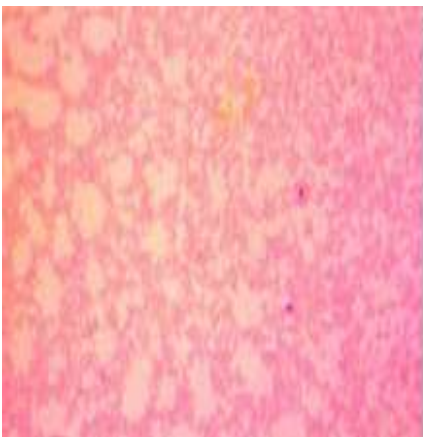

(c)

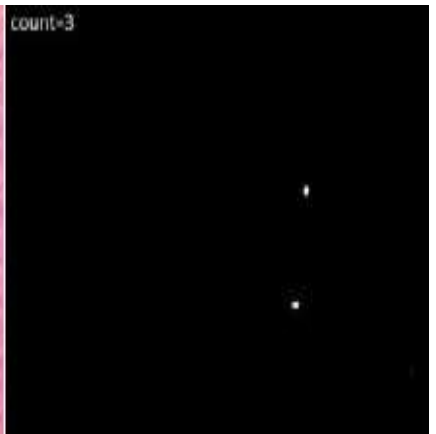

(d)
Fig 7: Images captured from the USB digital microscope. (a) Original image of ALL blood smear. (b) Binary image of the detected cells of the original image of ALL along with the count value. (c) Original image of normal blood smear.

(b) Binary image of the detected cells of the original image of normal blood smear along with the count value.

\section{CONCLUSION}

An efficient raspberry pi3 based hand held microscopic device is developed for identifying leukemia from the microscopic blood images. The developed hardware based system uses an image processing approach to screen the blood smear for the presence of leukemia. This method is reasonably faster than manual approach and it helps in consistent screening. The developed algorithm extracts WBC's from the microscopic blood image and discards other blood components. The count obtained from the hand held device is compared with the manual counting and machine counting approach. It ensures that the hand held device gives a closer value to the machine count than the manual count, with the accuracy of $94 \%$.

\section{ACKNOWLEDGEMENT}

We would like to thank Dr. Sushma Belurkar, Incharge, Hematology \& Clinical Pathology Kasturba Hospital, Manipal, for the valuable suggestions, help for acquiring microscopic blood images and encouragement throughout the project. We also thank Biomedical department, Manipal Institute of Technology, Manipal University, Manipal for providing excellent lab facilities to complete our work.

\section{REFERENCES}

[1]. M. Fadlee, A. Rashid and B. Woodward, "Blueooth Telemedicine Processor for Multichannel Biomedical Signal Transmission Via Mobile cellular Networks", IEEE Transactions on Information technology in Biomedicine, Vol 9, 2005, pp 35-43.

[2]. J. B. Pawley, "Handbook of Biological Confocal Microscopy", Springer Science and Business Media, LCC, 233 Spring Street, New York, NY 10013, USA, $3^{\text {rd }}$ Edition, 987-0-387-25921-5.

[3]. L. Deng, and M. S Poole, "Learning Through Telemedicine Networks", Hawaii, International Conference on System Science, IEEE Computer society, Vol 2, 2003, pp 736-742.
[4]. L. Faivdullah, F. Azahar, Z. Z. Htike, and W. Y. N. Naing, "Leukemia Detection From Blood Smears", Journal of Medical and Bioengineering, Vol 4, 2015, pp 488-491.

[5]. M. D. Joshi, A. H. Karode, and S. R. Suralkar, "Detection Of Acute Leukemia Using White Blood Cells Segmentation Based On Blood Samples", International Journal Of Electronics And Communication Engineering \& Technology, Vol 4, 2013, pp 148-153.

[6]. S. J. Mishra and A. P. Deshmukh, "Detection Of Leukemia Using Matlab", International Journal of Advanced Research in Electronics and Communication Engineering, Vol 4, 2015, pp 394-398.

[7]. M. Fatma and J. Sharma, "A Survey On Image Segmentation Techniques Used In Leukemia Detection", International Journal of Engineering Research and Applications, Vol 4, 2014, pp 66-71. 